\title{
Propiedades psicométricas de la Escala de Evaluación Funcional para el Tratamiento del Cáncer, versión próstata (FACT-P), en pacientes mexicanos
}

\author{
Psychometric properties of the Functional Assessment of Cancer Therapy, prostate \\ version (FACT-P), in Mexican patients
}

\author{
Marcos Espinoza-Bello ${ }^{1,2 *}$, Óscar Galindo-Vázquez², Miguel A. Jiménez-Ríos ${ }^{3}$, Abel Lerma ${ }^{4}$, \\ Nancy A. Acosta-Santos ${ }^{2}$, Abelardo A. Meneses-García ${ }^{5}$ y Juan J. Sánchez-Sosa ${ }^{1}$ \\ ${ }^{1}$ Facultad de Psicología, Universidad Nacional Autónoma de México, Ciudad de México; ${ }^{2}$ Servicio de Psicooncología, Instituto Nacional de \\ Cancerología (INCan), Ciudad de México; ${ }^{3}$ Departamento de Urología, INCan, Ciudad de México; ${ }^{4}$ Instituto de Ciencias de la Salud, Universidad \\ Autónoma del Estado de Hidalgo, San Agustín Tlaxiaca, Hidalgo; ${ }^{5}$ Dirección General, INCan, Ciudad de México. México
}

\begin{abstract}
Resumen
Antecedentes: El cáncer de próstata es la neoplasia más frecuente en los varones mexicanos, pero pocos estudios han abordado la calidad de vida en los pacientes con cáncer de próstata en México. Objetivo: Validar la Escala de Evaluación Funcional para el Tratamiento del Cáncer, versión próstata (FACT-P). Método: 201 pacientes de 49 a 90 años en cualquier etapa clínica, en tratamiento o seguimiento. Diseño de estudio: transversal no experimental. Se usaron el FACT-P, el Inventario de la Organización Europea para la Investigación y Tratamiento del cáncer y Calidad de Vida, y la Escala de Ansiedad y Depresión Hospitalaria. Se realizaron análisis factorial exploratorio y análisis factorial confirmatorio para evaluar el ajuste del modelo de los datos, mediante el método de máxima verosimilitud. Resultados: Se obtuvo un modelo de dos factores y dos indicadores que explicaron el $64.65 \%$ de la varianza, alfa de Cronbach 0.79 , correlaciones estadísticamente significativas, $r$ de Pearson de 0.146-0.716, $p<0.01$ y $p<0.05$. La escala discrimina los pacientes sin y con metástasis. Los principales índices del modelo confirmatorio sugieren un modelo estable y parsimonioso, con error próximo a cero, que se ajusta aceptablemente a los datos analizados. Conclusiones: La versión mexicana del FACT-P posee una confiabilidad y una validez adecuadas, similares a las de la original.
\end{abstract}

Palabras clave: Calidad de vida. Cáncer de próstata. FACT-P. México. Validación.

\begin{abstract}
Background: Prostate cancer is the most frequent neoplasm in Mexican men, the research literature contains few studies that address prostate cancer patients and quality of life in Mexico. Objective: To validate the Functional Assessment Cancer therapy (FACT-P) scale. Method: 201 males 49 to 90 years of age, at any clinical stage of prostate cancer, under treatment or follow-up participated. It's a non-experimental cross-sectional study. Patients were evaluated through the FACT-P jointly with the European Organization for Research and Treatment of Cancer Quality of Life and Hospital Anxiety and Depression Scale. Exploratory analysis examined the factorial structure, and confirmatory analysis to evaluate the adjustment of the exploratory model to the
\end{abstract}

\footnotetext{
Correspondencia:

${ }^{*}$ Marcos Espinoza-Bello

Avda. San Fernando, 22

Col. Sección XVI, Del. Tlalpan

Fecha de recepción: 15-12-2019

Cir Cir. 2020;88(6):745-752

C.P. 14080 , Ciudad de México, México

Fecha de aceptación: 12-08-2020

DOI: 10.24875/CIRU.19001755

Contents available at PubMed

E-mail: wolfx17@msn.com

www.cirugiaycirujanos.com

0009-7411/@ 2020 Academia Mexicana de Cirugía. Publicado por Permanyer. Este es un artículo open access bajo la licencia CC BY-NC-ND (http://creativecommons.org/licenses/by-nc-nd/4.0/).
} 
data. Results: A four-factor model that explained $64.65 \%$ of the variance, Cronbach's alpha 0.79 , and correlations were statistically significant, Pearson's $r$ of $0.146-0.716, p<0.01$ and $p<0.05$. Analyses also distinguished metastatic patients from nonmetastatic ones. The main indices of the confirmatory model were satisfactory for the adjustment of data and showed an estimate error close to zero. Conclusions: This Mexican version of FACT-P showed reliability and validity comparable to the original one.

Key words: Quality of life. Prostate cancer. FACT-P. Mexico. Validation study.

\section{Introducción}

Según los datos más recientes, se estima que la incidencia global del cáncer de próstata es de $1,276,106$ casos, con una mortalidad de 4.06 por cada 100,000 habitantes y una prevalencia estimada a 5 años de 3,724,658 casos, los cuales representan 96.73 por cada 100,000 habitantes'. En México, el Instituto Nacional de Estadística y Geografía informa que las defunciones causadas por cáncer han ido en aumento; en el año 2008 hubo 6.1 casos por cada 10 000 habitantes, y para el año 2017 se informan 6.8 casos por cada 10,000 habitantes. Las defunciones provocadas por tumores malignos se ubicaron en 84,142 casos, de los cuales los órganos genitourinarios representaron 19,408 casos (23.1\%). Por grupos de edad, el grupo más aquejado por dicho padecimiento son los que tienen una edad de 650 más años, con 41,088 fallecidos $(54.6 \%)^{2}$.

En México se identificaron 25,049 casos de cáncer de próstata, 13.1 por 100,000 habitantes, y hasta ese año se ubica como el tipo de neoplasia más frecuente en la población masculina. La cifra calculada de muertes fue de 6915 (8.3 por 100000 habitantes), lo cual representa la primera causa de muerte por cáncer en México. Finalmente, hay una prevalencia a 5 años de 55,565 personas (85.34 por cada 100000 habitantes) ${ }^{3}$.

La calidad de vida puede definirse como el grado en que se ve afectado el bienestar físico, emocional y social debido a una condición médica o a su tratamiento ${ }^{4}$. En el paciente con cáncer de próstata se ve afectada la calidad de vida por la enfermedad y los tratamientos médicos en diversas dimensiones, tanto físicas como psicológicas. Se han descrito los siguientes datos, por ejemplo, en cuanto a síntomas físicos: incontinencia urinaria (20\% en supervivencia, $65 \%$ en prostatectomía radical, $33 \%$ en radioterapia), urgencia al ir a orinar (52\% en supervivencia, $43 \%$ en prostatectomía radical, $75 \%$ en radioterapia), diarrea (13\% en supervivencia, $9 \%$ en prostatectomía radical, $20 \%$ en radioterapia), fatiga ( $7 \%$ en supervivencia, $8 \%$ en prostatectomía radical, $20 \%$ en radioterapia), disfunción eréctil (23\% en supervivencia, $76 \%$ en prostatectomía radical, $55 \%$ en radioterapia externa) y dolor (12\% en supervivencia, $18 \%$ en prostatectomía radical, $22 \%$ en radioterapia $)^{5}$.

En cuanto síntomas psicológicos, los hallazgos internacionales informan sensación de masculinidad disminuida ( $16 \%$ en supervivencia, $61 \%$ en prostatectomía radical, $49 \%$ en radioterapia externa), malestar emocional (16\% en supervivencia, $9 \%$ en prostatectomía radical, $12 \%$ en radioterapia externa $)^{5}$, depresión (17\% en pretratamiento, $15 \%$ en tratamiento y $18 \%$ en postratamiento) y ansiedad (27\% en pretratamiento, $15 \%$ en tratamiento y $18 \%$ en postratamiento) ${ }^{6}$.

Dados los resultados anteriores, resulta necesario evaluar de manera precisa las dimensiones de calidad de vida, y además, en población mexicana con cáncer de próstata hay poca investigación al respecto ${ }^{7,8}$. Hasta el momento no se ha identificado una escala diseñada o validada para este grupo de pacientes. El hecho de no contar con un instrumento específico limita el conocimiento preciso de la calidad de vida y de las diferencias culturales que puedan encontrarse con otras poblaciones.

En este contexto, se validó la Escala de Evaluación Funcional para el Tratamiento del Cáncer (Functional Assessment of Cancer Therapy), versión próstata $(\text { FACT-P })^{9}$, que ha mostrado su utilidad en diversos entornos culturales ${ }^{10,11}$ y ha revelado niveles de consistencia interna en que van de 0.687 en el caso de la escala china ${ }^{11}$ a 0.880 para la versión coreana ${ }^{10}$. La escala también ha mostrado que es capaz de discriminar a los pacientes con y sin metástasis ${ }^{9}$, todo lo cual hace relevante su posible uso en diversos países. La FACT-P es una escala autoaplicada conformada por 39 reactivos en su versión original, que presenta una consistencia interna de $0.87-0.89$ y para la subescala de cáncer de próstata un alfa de 0.65-0.699.

\section{Método}

\section{Participantes}

Participaron 201 pacientes con cáncer de próstata reclutados en el Instituto Nacional de Cancerología 
(INCan), Ciudad de México. Los criterios de inclusión fueron: diagnóstico oncológico de cáncer de próstata, en tratamiento oncológico (cualquier tipo o combinación de estos) o periodo de seguimiento, cualquier etapa clínica, y que supieran leer y escribir. Los criterios de exclusión fueron: pacientes con deficiencias cognitivas, pacientes con deficiencias auditivas y pacientes con deficiencias visuales. Los criterios de eliminación fueron: que el paciente decidiera no seguir participando o que no hubiera completado las respuestas a los instrumentos. La media de edad fue de 68.5 años (49-90) y la tabla 1 muestra los datos sociodemográficos de los participantes. El presente estudio fue aprobado previamente por el Comité de Ética e Investigación del INCan.

\section{Instrumentos}

La FACT-P es una escala multidimensional autoaplicada conformada por 39 reactivos, de los cuales 12 son específicos para cáncer de próstata, que mide dolor, función urinaria, función sexual, aspectos sociales y emocionales, con coeficientes de consistencia interna de $0.65-0.69^{12}$ y que se aplica de forma conjunta con el FACT-G, con un puntaje alfa de consistencia interna de 0.87-0.8913.

EI EORTC QLQ-C30 (European Organization for Research and Treatment of Cancer Quality of Life Questionnaire) validado en México ${ }^{14}$ contiene 30 reactivos con cuatro escalas de dominio (físico, rol emocional, social y cognitivo); dos reactivos evalúan la calidad de vida global, y además tiene tres escalas de síntomas (fatiga, dolor y vómitos), con coeficientes de consistencia interna de 0.60-0.7015.

La Escala de Ansiedad y Depresión Hospitalaria $(E A D H)^{16}$, ya validada en México ${ }^{17}$, es un instrumento que mide sintomatología ansiosa y depresiva, con 12 reactivos en su versión mexicana, con consistencia interna global (alfa $=0.86$ ) y coeficientes para cada subescala con un valor de $0.79-0.80$, y muestra un $48.04 \%$ de la varianza explicada.

Se agregó una cédula de identificación del participante, la cual recabó variables clínicas y sociodemográficas del Sistema INCan, conservando todos los criterios éticos y de confidencialidad.

\section{Adaptación cultural}

Se aplicó una prueba piloto del cuestionario a 30 pacientes mexicanos con la versión en español de la FACT-P para explorar posibles expresiones confusas o interpretables de más de una forma y para asegurar que los pacientes comprendieran el contenido de los reactivos ${ }^{18}$. Se aplicó un formato de registro de caso que incluyó un cuestionario específico con el fin de cubrir la comprensión de las instrucciones, de cada reactivo y de las opciones de respuesta. Los datos recabados indicaron que el $16.7 \%$ de participantes tuvieron problemas de comprensión en un reactivo, cercano al criterio del $20 \%$ que se considera para modificar el reactivo. A partir de estos datos se llevó a cabo un proceso de «jueceo» para valorar la estructura de los reactivos, buscando información, certeza, juicio u opinión para revisar la versión en español del reactivo ${ }^{19}$. Al reactivo en inglés «l am able to feel like a man» se podía agregar la palabra «un», por lo quedaría como "Soy capaz de sentir como un hombre». Esta observación se envió a un grupo de psicólogos titulados con grado mínimo de maestría junto con una plantilla de instrucciones y no hubo un consenso respecto al cambio, por lo que se empleó el reactivo con la traducción literal.

\section{Análisis}

Se realizaron análisis de tendencia central mediante la prueba de Kolmogórov-Smirnov, análisis descriptivos con mediana y moda para la estadística inferencial del FACT-P. Los criterios utilizados para el análisis fueron los siguientes: no se asignaron cargas factoriales mínimas, no se tomó un mínimo de reactivos por factor y se tomó el coeficiente de consistencia interna por cada factor con un alfa de Cronbach $>0.60$. Para la relación entre FACT-P, EORTC QLQ30 y HADS se empleó la correlación de Pearson por el nivel de intervalo de las variables con $p<0.05$. Además, se realizaron análisis de consistencia interna y análisis factorial exploratorio mediante rotación varimax, ya que se identificaron correlaciones bajas y medianas entre reactivos ${ }^{20}$. Para los análisis anteriores se empleó la versión 24 del software IBM SPSS $^{\circledR}$.

Adicionalmente se realizó una comparación de niveles de calidad de vida empleando las subescalas del FACT-P entre pacientes sin y con metástasis. Para ello se realizó la prueba $U$ de Mann Whitney. A partir de los resultados del modelo exploratorio se efectuó un análisis factorial confirmatorio con el método de máxima verosimilitud usando el programa estadístico AMOS (versión 23.0), para estimar su ajuste a los datos mediante los índices siguientes: para el ajuste global, el valor de ji al cuadrado 
Tabla 1. Datos demográficos de los participantes para validación del FACT-P

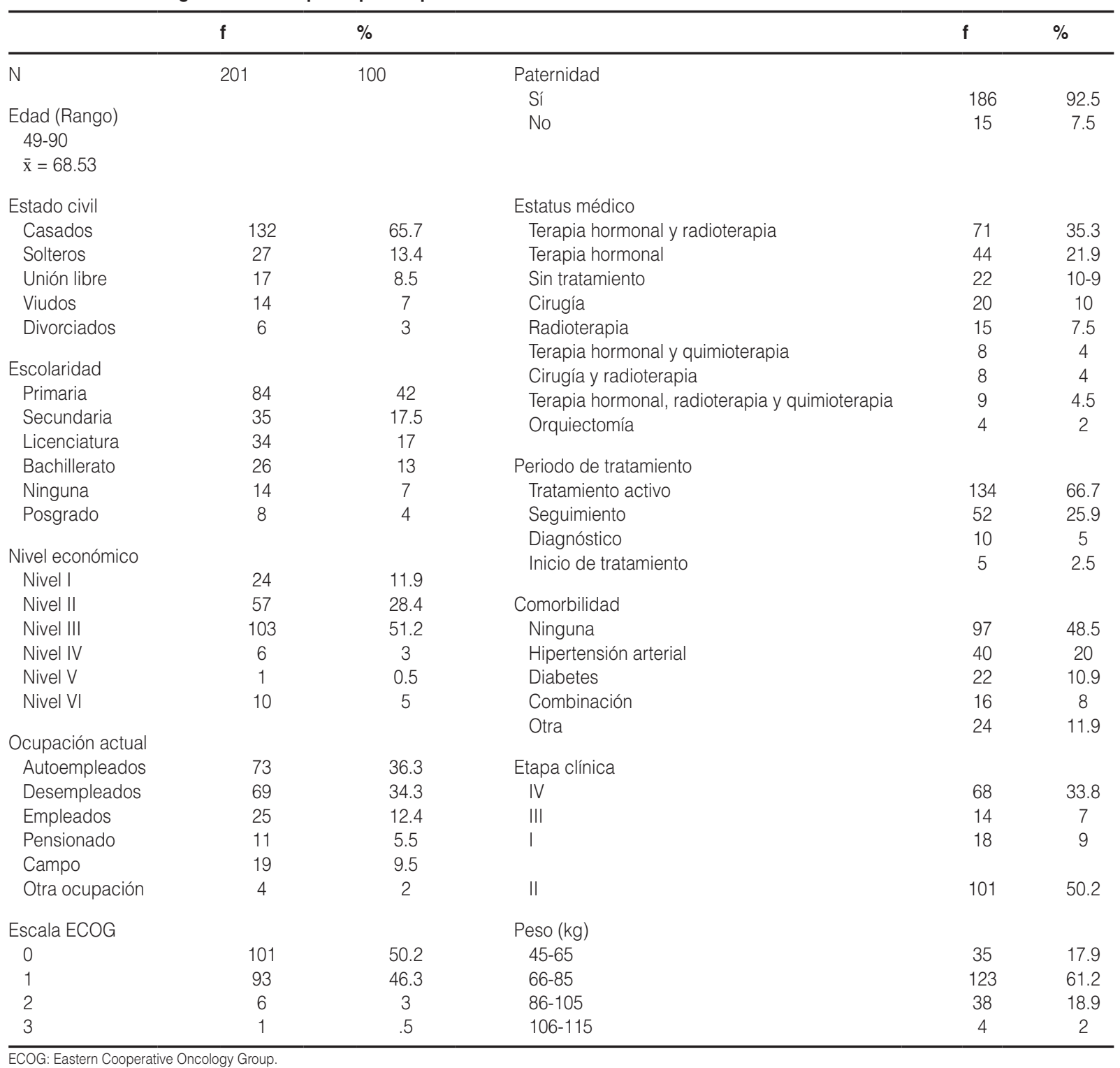

absoluto y luego la razón ji al cuadrado/grados de libertad para confirmar si existen errores en las varianzas y covarianzas nulos, y si se ajusta a muestras grandes $(n \geq 200)$ como recomiendan varios expertos $^{21,22}$.

Se estimó el ajuste global contra un modelo nulo mediante el índice Comparative Fit Index (CFI) y el índice de Tucker-Lewis (TLI), que «penaliza» la complejidad y el ajuste del modelo a los datos ${ }^{23}$.

La parsimonia y el equilibrio del modelo se estimaron mediante el TLI, al tiempo que la bondad de ajuste mediante Goodness of Fit Index (GFI), Adjusted Goodness of Fit Index (AGFI) y la raíz del residuo cuadrático medio de aproximación (RMSEA). Por último, se buscó la ratificación de la bondad de ajuste mediante el cálculo del índice de error residual medio (RMR ${ }^{23-25}$.

\section{Resultados}

\section{Estructura factorial}

La escala FACT-P obtuvo en la prueba de esfericidad de Bartlett una $\mathrm{p}=0.001$ y un índice $\mathrm{KMO}=0.774$. Las correlaciones entre reactivos fueron entre 0.145 y 0.645 . Se identificó un alfa de Cronbach (consistencia interna) de 0.79 con la totalidad de los reactivos. el análisis factorial exploratorio arrojó un modelo de dos factores (dolor alfa $=0.81$ y función urinaria 
Tabla 2. Factores rotados para el FACT-P, ordenados según su carga factorial

\begin{tabular}{|c|c|c|c|c|c|c|c|}
\hline \multirow[t]{2}{*}{ Reactivos subescala FACT-P } & \multicolumn{4}{|c|}{ Componente } & \multicolumn{3}{|c|}{ Alfa 12 reactivos $(\alpha=0.79)$} \\
\hline & 1 & 2 & 3 & 4 & $\begin{array}{l}\text { Varianza } \\
\text { explicada }\end{array}$ & Media DE & $\begin{array}{l}\text { Alfa de } \\
\text { Cronbach }\end{array}$ \\
\hline & & & & & $64.65 \%$ & & \\
\hline Subescala dolor & & & & & & $11.68 \pm 3.75$ & $\alpha=0.81$ \\
\hline P1 Tengo dolores que me molestan & 0.913 & 0.128 & 0.062 & 0.046 & & & \\
\hline P2 Tengo dolor en ciertas partes del cuerpo & 0.904 & 0.154 & 0.125 & -0.033 & & & \\
\hline P3 El dolor me impide hacer las cosas que quiero hacer & 0.751 & 0.246 & 0.145 & 0.133 & & & \\
\hline P6 Tengo problemas con el estreñimiento & 0.420 & 0.257 & -0.092 & 0.141 & & & \\
\hline Subescala función urinaria & & & & & & $12.03 \pm 3.16$ & $\alpha=0.70$ \\
\hline BL2 Orino más frecuentemente de lo usual & 0.133 & 0.788 & 0.113 & -0.140 & & & \\
\hline P7 Tengo dificultad al orinar & 0.103 & 0.771 & 0.026 & 0.115 & & & \\
\hline P8 Mis problemas con el orinar limitan mis actividades & 0.330 & 0.710 & 0.025 & 0.069 & & & \\
\hline C2 Estoy bajando de peso & 0.327 & 0.391 & -0.186 & 0.374 & & & \\
\hline Subescala función sexual & & & & & & $2.8 \pm 1.8$ & $\alpha=0.48$ \\
\hline BL5 Soy capaz de tener y mantener una erección & 0.109 & 0.118 & 0.807 & -0.124 & & & \\
\hline P5 Soy capaz de sentir como un hombre & 0.005 & -0.008 & 0.722 & 0.426 & & & \\
\hline Subescala funcionalidad & & & & & & $4.9 \pm 1.7$ & $\alpha=0.50$ \\
\hline C6 Tengo buen apetito & 0.005 & 0.063 & 0.042 & 0.830 & & & \\
\hline P4 Estoy satisfecho con el alivio que tengo por el momento & 0.402 & -0.109 & 0.425 & 0.568 & & & \\
\hline
\end{tabular}

DE: Desviación estándar.

Análisis factorial de cuatro factores, Kaiser-Meyer-Olkin 0.774 , Batlett $p>0.001$

alfa $=0.70$ ) con dos indicadores (función sexual alfa $=$ 0.48 y funcionalidad alfa $=0.50$ ) que explicó el $65.7 \%$ de la varianza total, con estimadores totales $31.44 \pm$ 7.36 (media y desviación estándar), estos factores e indicadores son equivalentes a los informados en otros estudios (Tabla 2).

\section{Discriminación entre pacientes con y sin metástasis}

Para evaluar la capacidad del FACT-P para diferenciar la calidad de vida de los pacientes con y sin metástasis, una vez realizados los análisis existen diferencias estadísticamente significativas en la puntuación total del instrumento $(p \leq 0.001)$. El grupo que tiene peor calidad de vida es el de los pacientes con metástasis en las siguientes subescalas: subescala de cáncer de próstata $(p=0.009)$, índice de resultados en los ensayos ( $p \leq 0.001$ ), estado físico general de salud ( $p \leq 0.001$ ) y capacidad de funcionamiento personal ( $p=0.006$ ); $\sin$ embargo, no hay diferencias significativas en las subescalas de ambiente familiar y social $(p=0.691)$ y de estado emocional $(p=0.565)$.

\section{Análisis confirmatorio}

En la figura 1 se observan el modelo con la estructura final de cuatro factores, los estimadores calculados para cada factor y las correlaciones entre ellos, así como la varianza explicada por cada reactivo. En la parte inferior se indican los índices más relevantes, como son los de ajuste comparativo relativos (CFI, TLI y AGFI), cuyos valores son ideales (>0.90) y corroboran un modelo aceptable y parsimonioso, comparado contra el nulo ${ }^{22}$. El ajuste general se observa excelente en el valor de ji al cuadrado ajustado a 48 grados de libertad (Chi-square Mean Index/Degree of 


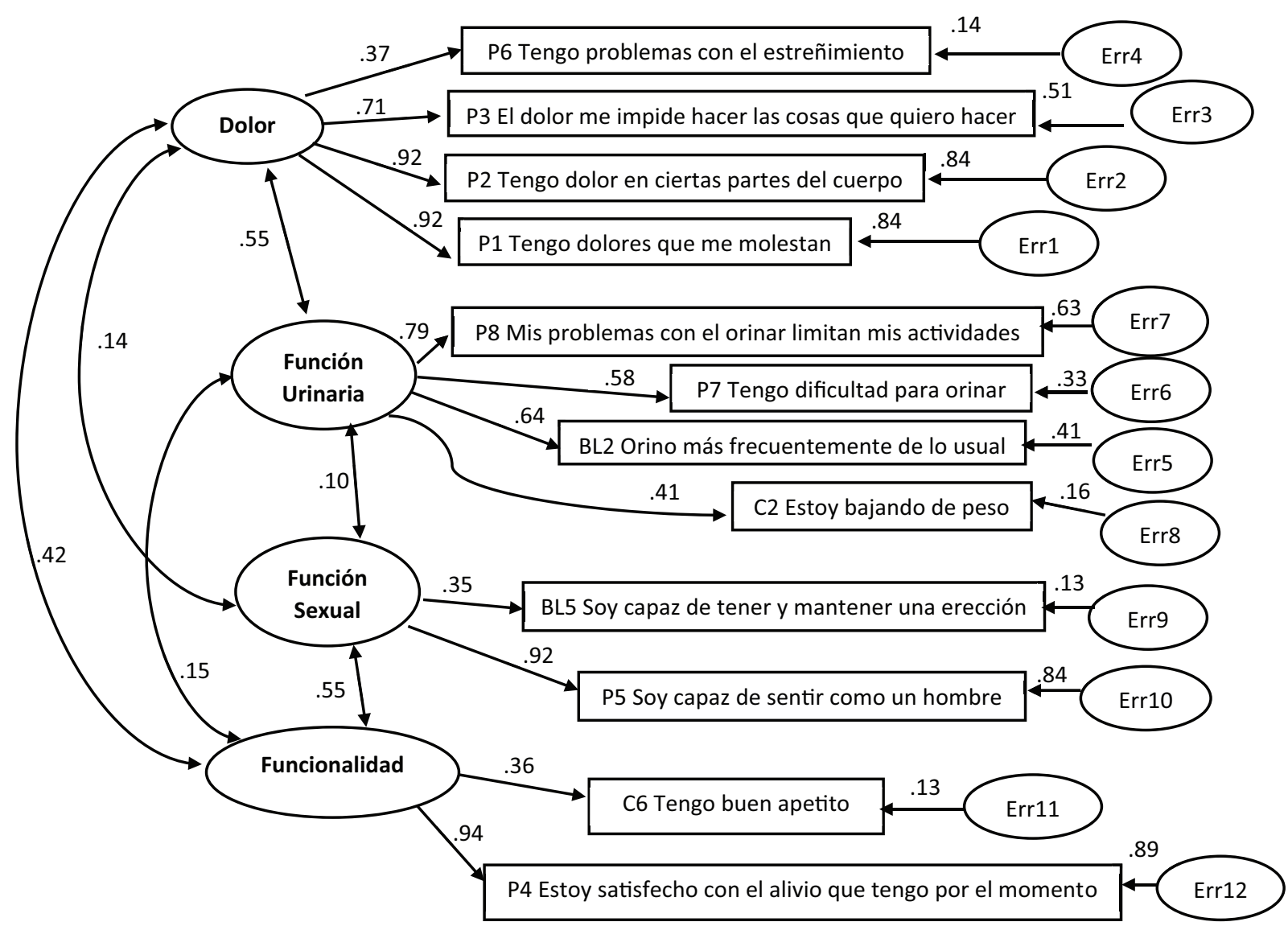

Figura 1. Modelo de análisis factorial confirmatorio de cuatro factores para la FACT-P en pacientes con cáncer de próstata $(N=201)$. Ji al cuadrado $=77.43,48$ grados de libertad, $0=005, C M I N / g I=1.613, R M R=0.078, G F I=0.941, A G F I=0.904, T L I=0.944, C F I=0.960$, RMSEA $=0.055$ (0.031-0.077), Hoelter (adecuación muestral, $p=01, n=191$ ).

Freedom $[\mathrm{CMIN}] / \mathrm{gl}=1.613$ ), lo que confirma un excelente ajuste absoluto del modelo a los datos observados $^{25}$.

El índice RMR está muy cercano a cero y es $<0.08$ $(\mathrm{RMR}=0.078)$, lo que indica una diferencia casi nula entre la matriz de covarianza observada y la predicha, y podemos asumir que la discrepancia entre el modelo propuesto y los datos reales es casi nula o muy baja ${ }^{21,24,25}$.

El valor del índice RMSEA (radical del error de aproximación, o raíz cuadrada media de la razón entre el parámetro no central ajustado por los grados de libertad) es casi cero (RMSEA $=0.055 ; 0.031-0.077$ ) e indica casi nulidad en el error del modelo25.

En la prueba de Hoelter ( $n=191, p=0.01$ ), el tamaño de muestra teórico no supera el utilizado, por lo que podemos afirmar que el modelo es correcto y se acepta la hipótesis (probabilidad de un error alfa de 0.01 ) de ser una muestra suficiente para los análi$\operatorname{sis}^{24,25}$ (Fig. 1).
Se realizó una validez concurrente del FACT-P empleando los instrumentos EORTC QLQ 30 y HADS, y las correlaciones fueron positivas y estadísticamente significativas ( $r$ de Pearson de 0.146-0.716, $p<0.01$ y $p<0.05$ ) entre las subescalas, por ejemplo salud global, función física y roles funcional, emocional, cognitivo y social. Por otro lado, se identificaron correlaciones negativas entre FACT-P, EORTC QLQ 30 y HADS, Io que indica una relación inversamente proporcional $(r$ de Pearson de -0.206 a $0.0744, p<0.01$ ) en fatiga, náuseas, dolor, sintomatología de ansiedad, depresión y puntuación total HADS (Tabla 3).

\section{Discusión}

EI FACT-P está considerado como una de las mejores herramientas para medir la calidad de vida en los pacientes con cáncer de próstata ${ }^{26}$, y las validaciones en otros países así lo muestran ${ }^{10,11}$, así como los resultados adecuados que mostró en los pacientes mexicanos en este estudio. 
Tabla 3. Coeficientes de correlación del FACT-P versión mexicana con EORTC QLQ-30 y HADS

\begin{tabular}{|c|c|c|c|c|c|c|c|c|c|c|c|c|}
\hline \multirow{2}{*}{$\begin{array}{l}\text { Subescala/ } \\
\text { escala total } \\
\text { FACT-P }\end{array}$} & \multicolumn{9}{|c|}{ EORTC QLQ-30 } & \multicolumn{3}{|c|}{ HADS } \\
\hline & $\begin{array}{l}\text { Salud } \\
\text { global }\end{array}$ & $\begin{array}{l}\text { Función } \\
\text { física }\end{array}$ & $\begin{array}{c}\text { Rol } \\
\text { funcional }\end{array}$ & Emocional & Cognitivo & Social & Fatiga & Náusea & Dolor & $\begin{array}{c}\text { HADS } \\
\text { ansiedad }\end{array}$ & $\begin{array}{c}\text { HADS } \\
\text { depresión }\end{array}$ & $\begin{array}{l}\text { HADS } \\
\text { total }\end{array}$ \\
\hline PCS & $0.581^{\dagger}$ & $0.716^{+}$ & $0.641^{\dagger}$ & $0.440^{\dagger}$ & $0.384^{\dagger}$ & $0.484^{\dagger}$ & $-0.679^{+}$ & $-0.498^{\dagger}$ & $-0.764^{\dagger}$ & $-0.445^{\dagger}$ & $-0.559^{\dagger}$ & $0.581^{\dagger}$ \\
\hline TOI & $0.659^{\dagger}$ & $0.720^{+}$ & $0.664^{+}$ & $0.486^{+}$ & $0.407^{\dagger}$ & $0.533^{+}$ & $-0.699^{\dagger}$ & $-0.522^{\dagger}$ & $-0.744^{+}$ & $-0.524^{\dagger}$ & $-0.634^{\dagger}$ & $0.659^{\dagger}$ \\
\hline PWB & $0.612^{\dagger}$ & $0.706^{\dagger}$ & $0.658^{\dagger}$ & $0.512^{\dagger}$ & $0.377^{\dagger}$ & $0.546^{\dagger}$ & $-0.774^{\dagger}$ & $-0.622^{\dagger}$ & $-0.753^{\dagger}$ & $-0.509^{\dagger}$ & $-0.563^{\dagger}$ & $0.612^{\dagger}$ \\
\hline SWB & $0.202^{\dagger}$ & & & & & $0.146^{*}$ & & & & & & $0.202^{\dagger}$ \\
\hline EWB & $0.365^{\dagger}$ & $0.259^{+}$ & $0.254^{+}$ & $0.579^{+}$ & $0.308^{+}$ & $0.305^{+}$ & $-0.342^{\dagger}$ & $-0.244^{\dagger}$ & $-0.268^{+}$ & $-0.545^{\dagger}$ & $-0.389^{\dagger}$ & $0.365^{\dagger}$ \\
\hline FWB & $0.496^{\dagger}$ & $0.406^{\dagger}$ & $0.391^{\dagger}$ & $0.288^{\dagger}$ & $0.275^{\dagger}$ & $0.330^{+}$ & $-0.320^{+}$ & $-0.206^{\dagger}$ & $-0.365^{\dagger}$ & $-0.388^{\dagger}$ & $-0.501^{\dagger}$ & $0.496^{\dagger}$ \\
\hline
\end{tabular}

EWB: estado emocional; FWB: funcionamiento personal; PCS: escala de próstata; PWB: estado físico; SWB: ambiente social y familiar; TOI: índice de resultados en los ensayos (en la sumatoria se emplean solo estado físico, funcionamiento personal y escala de próstata).

*La correlación es significativa al nivel 0.05 .

†La correlación es significativa al nivel 0.01 .

La evaluación de la calidad de vida en los pacientes con cáncer de próstata es de gran relevancia, ya que es posible documentar de manera rápida y confiable cómo percibe la persona el impacto que tienen en su vida la enfermedad y los tratamientos recibidos. Debido al deterioro que suelen presentar estos pacientes en variables tanto físicas como psicológicas, es necesario establecer de manera razonablemente certera cómo se ven afectadas dichas variables ${ }^{27}$.

Para el análisis, el número de participantes resultó adecuado. Además, en el análisis factorial según lo confirmaron la prueba de esfericidad de Bartlett y la prueba Kaiser-Meyer-Olkin y la rotación varimax, se decidió que las correlaciones entre reactivos se ubican en los criterios de pequeñas o medianas.

Adicionalmente, es de destacar que la FACT-P también discrimina de manera adecuada a los pacientes con y sin metástasis, al igual que lo hace la escala original, considerando solo aspectos físicos. En contaste, no discrimina las subescalas de ambiente familiar y social y de estado físico, pero podría considerarse que estas variables no necesariamente tienen relación directa con la metástasis en sentido estricto.

Los presentes resultados respecto a la FACT-P concuerdan con lo informado por la literatura internacional de investigación ${ }^{9,12}$, con una estructura cuyas subescalas miden dolor, función urinaria, función sexual, aspectos sociales y emocionales relacionados con el cáncer de próstata. Sin embargo, el reactivo «Estoy bajando de peso", por su carga factorial, se relacionó con la función urinaria (Tabla 2). En teoría, el reactivo no tendría una relación directa con el resto de las preguntas de función urinaria, pero los pacientes comprendieron que bajar de peso y los problemas para orinar podrían estar estrechamente relacionados según otros datos previamente informados ${ }^{28}$. Cabe señalar que el estudio sobre dichos datos se basó en pacientes obsesos con problemas en la función urinaria, y el estudio indicó que menores problemas para orinar estarían relacionados con un peso más adecuado. En virtud de lo anterior, se decidió dejar este reactivo en esa posición, a reserva de que futuros estudios observen el comportamiento de dicho reactivo para facilitar una decisión al respecto, es decir, sea que se coloque en otro factor o que se elimine de la escala.

Se considera necesario que futuras investigaciones puedan evaluar la estabilidad del instrumento a lo largo del tiempo, y también se sugiere que se aplique a pacientes con reciente diagnóstico y a pacientes en seguimiento para un abordaje oportuno multidisciplinario en calidad de vida, además de comparar los resultados con poblaciones distintas.

\section{Conclusiones}

La escala FACT-P es un instrumento confiable y válido, estable y parsimonioso para medir la calidad de vida de los pacientes con cáncer de próstata en México.

\section{Agradecimientos}

Los autores agradecen a la doctora Carmen Lizette Gálvez Hernández por su colaboración en la 
redacción del protocolo de esta investigación y la revisión del pilotaje del instrumento.

\section{Conflicto de intereses}

Los autores de este trabajo declaran no tener ningún conflicto de intereses.

\section{Financiamiento}

Consejo Nacional de Ciencia y Tecnología (CONAСуT), número de beca 697234 .

\section{Responsabilidades éticas}

Protección de personas y animales. Los autores declaran que para esta investigación no se han realizado experimentos en seres humanos ni en animales.

Confidencialidad de los datos. Los autores declaran que han seguido los protocolos de su centro de trabajo sobre la publicación de datos de pacientes.

Derecho a la privacidad y consentimiento informado. Los autores han obtenido el consentimiento informado de los pacientes y/o sujetos referidos en el artículo. Este documento obra en poder del autor de correspondencia.

\section{Bibliografía}

1. Bray F, Ferlay J, Soerjomataram I, Siegel RL, Torre LA, Jemal A. Global cancer statistics 2018: GLOBOCAN estimates of incidence and mortality worldwide for 36 cancers in 185 countries. CA Cancer J Clin. 2018;68:394-424.

2. Instituto Nacional de Estadística y Geografía. Características de las defunciones registradas en México durante 2017. México; 2018. p. 1-60. Report No.: 525/18. [Consultado el 29 de julio de 2019] Disponible en: https://www.inegi.org.mx/contenidos/saladeprensa/boletines/2018/EstSociodemo/DEFUNCIONES2017.pdf

3. Ferlay JE, Lam F, Colombet M, Mery L, Pineros M, Znaor A, et al. Global cancer observatory: cancer today. 2019. [Consultado el 4 de agosto de 2019]. Disponible en: http://gco.iarc.fr/today/home

4. Cella D. Measuring quality of life in palliative care. Semin Oncol. 1995;22(2 Suppl 3):73-81.

5. Stam M-A van, Aaronson NK, Bosch JLHR, Kieffer JM, Zyp JRN van de $\checkmark$ van, Tillier $C N$, et al. Patient-reported Outcomes Following Treatment of Localised Prostate Cancer and Their Association with Regret About Treatment Choices. Eur Urol Oncol. 2020;3:21-31.

6. Watts S, Leydon G, Birch B, Prescott P, Lai L, Eardley S, et al. Depression and anxiety in prostate cancer: a systematic review and meta-analysis of prevalence rates. BMJ Open. 2014;4:e003901.

7. Sierra-Guerra KL, Viveros-Contreras C, Martínez-Carrillo G, Hernández-León O, Caballero-Ambriz G. Calidad de vida en pacientes con cáncer de próstata, operados de prostatectomía radical laparoscópica. Rev Mex Urol. 2014;74(3):133-140.
8. Vázquez L, Camarena H, Martínez JA, Hernández V. Estudio comparativo de calidad de vida en pacientes con cáncer de próstata con diferentes tratamientos iniciales. Rev Mex Urol. 2009;69:143-6.

9. Esper P, Mo F, Chodak G, Sinner M, Cella D, Pienta K. Measuring quality of life in men with prostate cancer using the Functional Assessment of Cancer Therapy-Prostate Instrument. Adult Urol. 1997;50:920-8.

10. Hong JH, Jeon SS, Lee HM, Choi YH, Kim S, Choi HY. The Functional Assessment of Cancer Therapy-Prostate (FACT-P) scales in men with prostate cancer: reliability and validity of the Korean version. J Korean Med Sci. 2006;21:295-9.

11. Wong $\mathrm{CKH}$, Choi EPH, Tsu JHL, Ho BSH, Ng ATL, Chin WY, et al. Psychometric properties of Functional Assessment of Cancer Therapy-Prostate (FACT-P) in Chinese patients with prostate cancer. Qual Life Res. 2015;24:2397-402.

12. Chu D, Popovic M, Chow E, Cella D, Beaumont JL, Lam H, et al. Development, characteristics and validity of the EORTC QLQ-PR25 and the FACT-P for assessment of quality of life in prostate cancer patients. J Comp Eff Res. 2014;3:523-31.

13. Cella D, Tulsky DS, Gray G, Sarafian B, Linn E, Bonomi A, et al. The Functional Assessment of Cancer Therapy scale: development and validation of the general measure. J Clin Oncol. 1993;11:570-9.

14. Oñate-Ocaña LF, Alcántara-Pilar A, Vilar-Compte D, García-Hubard G, Rojas-Castillo E, Alvarado-Aguilar S, et al. Validation of the Mexican Spanish Version of the EORTC C30 and STO22 questionnaires for the evaluation of health-related quality of life in patients with gastric cancer. Ann Surg Oncol. 2009;16:88-95.

15. Aaronson NK, Ahmedzai S, Bergman B, Bullinger M, Cull A, Duez NJ, et al. The European Organization for Research and Treatment of Cancer QLQ-C30: a quality-of-life instrument for use in international clinical trials in oncology. J Natl Cancer Inst. 1993;85:365-76.

16. Zigmond AS, Snaith RP. The Hospital Anxiety and Depression Scale. Acta Psychiatr Scand. 1983;67:361-70.

17. Galindo O, Benjet $\mathrm{C}$, Juárez $\mathrm{F}$, Rojas E, Riveros A, Aguilar JL, et al. Propiedades psicométricas de la Escala Hospitalaria de Ansiedad y Depresión (HADS) en una población de pacientes oncológicos mexicanos. Salud Ment. 2015;38:253-8.

18. Mora J, Bautista N, Natera G, Pedersen D. Adaptación cultural de instrumentos de medida sobre estigma y enfermedad mental en la Ciudad de México. Salud Ment. 2013;36:9-18.

19. Skjong $\mathrm{R}$, Wentworth $\mathrm{BH}$. Expert judgment and risk perception. The Eleventh International Offshore and Polar Engineering Conference. Mountain View, CA: International Society of Offshore and Polar Engineers; 2001. pp. 1-8.

20. Reyes I, García L. Procedimiento de validación psicométrica culturalmente relevante: un ejemplo. En: Rivera S, Díaz R, Sánchez R, Reyes I, editores. La psicología social en México. México: Asociación Mexicana de Psicología Social; 2008. p. 625-36.

21. Ullman JB. Structural equation modeling: reviewing the basics and moving forward. J Pers Assess. 2006;87:35-50.

22. Hu L, Bentler PM. Fit indices in covariance structure modeling: sensitivity to underparameterized model misspecification. Psychol Methods. 1998;3:424-53.

23. Kline RB. Principles and practice of structural equation modeling. 4th ed. New York: The Guilford Press; 2011. [Consultado el 27 de agosto de 2019]. Disponible en: https://www.guilford.com/books/Principles-and-Practice-of-Structural-Equation-Modeling/Rex-Kline/9781462523344

24. George D, Mailery M. Using SPSS for Windows step by step: a simple guide and reference. Boston: Aliyn \& Bacon; 2001.

25. Byrne BM. Structural equation modeling with AMOS: basic concepts, applications, and programming. New York, NY, US: Routledge/Taylor \& Francis Group; 2010.

26. Morris C, Gibbons E, Fitzpatrick R. A structured review of patient-reported outcome measures (PROMs) for prostate cancer. University of Oxford Web site; 2009. [Consultado el 25 de marzo de 2016]. Disponible en: http://phi.uhce.ox.ac.uk/pdf/CancerReviews/PROMs_Oxford_Prostate\%20Cancer_012011.pdf.

27. Harrington S, Lee J, Colon G, Alappattu M. Oncology Section EDGE Task Force on Prostate Cancer: a systematic review of outcome measures for health-related quality of life. Rehabil Oncol Am Phys Ther Assoc Oncol Sect. 2016;34:27-35.

28. Subak LL, Wing R, West DS, Franklin F, Vittinghoff E, Creasman JM, et al. Weight loss to treat urinary incontinence in overweight and obese women. N Engl J Med. 2009;360:481-90. 